\title{
EFFECTS OF CONTROLLED DEFECTS ON THE VORTEX-SOLID MELTING TRANSITION OF Y-Ba-Cu-O SINGLE CRYSTALS
}

W. JLANG, N.-C. YEI, D. S. REED, U. KRIPLANI, T. A. TOMBRELLO AND A. P. RICE Department of Physics, California Institute of Technology

Pasadena, CA 91125

\section{F. HOLTZBERG}

IBM Research Divisjon, Thomas J. Watson Research Center

Yorktown Heights, NY 10598

\section{ABSTRACT}

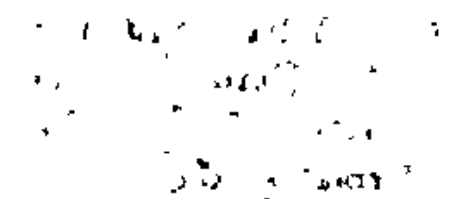

We report systematic studies of the dc transport properties in proton-irradiated Y-Ba$\mathrm{Cu}-\mathrm{O}$ single crystals. We find that the onset of vortex dissipation in moderately irradiated samples is associated with the occurrence of a second-order vortex-solid melting transition. In addition, the decreasing zero-field transition temperature and increasing critical current density with the increasing defects reveal the effects of disorder on reducing the electron mean-free-path and on increasing the pinning density.

\section{INTRODUCTION}

Irradiation is a standard technique to introduce defects into materials systematically. Although many investigations have studied the effect of irradiation on the critical current densities of bigh temperature superconductors (HTS), most observations consisted of indactive measurements of the sample magnetization $[1,2]$. In this paper, we present systematic studies of the dc current-voltage characteristics of a proton-irradiated $\mathrm{Y}-\mathrm{Ba}-\mathrm{Cu}-\mathrm{O}$ single crystal. The vortex transport properties of the sample after irradiations follow the same critical scaling behavior as that reported for a sample without irradiation $[3,4]$. In addition, the following effects are observed with increasing degrees of disorder: the zerofield transition temperature $T_{\infty 0}$ is reduced, the normal state resistivity $\rho$ is increased, and the critical current density $J_{c}$ is enhanced.

\section{EXPERIMENTAL}

The parent rample is a well characterized twinned $\mathrm{Y}-\mathrm{Ba}-\mathrm{Cu}-\mathrm{O}$ single crystal $[3,5]$ with $T_{\infty 0}=92.95 \mathrm{~K}$ and sample dimensions $0.90 \times 0.65 \times 0.021 \mathrm{~mm}^{3}$. 3-.Mev proton irradiations are performed at room temperature using a tandem Van de Graaff accelerator. The beam orientation is parallel to the $c$-axis of the sample. The sample is irradiated twice with a fluence of $5 \times 10^{13}$ protons $/ \mathrm{cm}^{2}$ each. In the following we will denote the sample before the irradiation, after the first irradiation, and after the second irradiation as Samples $A$, $B$, and $\mathrm{C}$, respectively. Since the range of $3-\mathrm{Mev}$ protons in $\mathrm{Y}-\mathrm{Ba}-\mathrm{Cu}-\mathrm{O}$ is about $45 \mu \mathrm{m}$, greater than the crystal thickness, all incident protons can pass completely through the sample. The defects created by $3-\mathrm{Mev}$ protons are randomly distributed small clusters. $A$ simple calculation based on collision theory shows that the density of the defects is about $1.1 \times 10^{19} / \mathrm{cm}^{3}$ for a fluence of $5 \times 10^{15} / \mathrm{cm}^{2}$. Since the pinning strength of the clusters is much weaker than that of the twin boundaries, we can observe gradual changes in the transport properties by controlling the density of the defects. 
The transport measurements are carried out using the standard four-probe method. The dc magnetic field $\vec{H}$ is applied along the sample $c$-axis, and the current is applied in the $a b$ plane with $\vec{J} \perp \vec{H}$. More details of the experimental techniques and sample characterizations have been given in Ref. [5].

\section{RESULTS AND ANALYSIS}

The electric field $(E)$ versus dc current density $(J)$ isotherms for $\mathrm{H}$ ranging from 0 to $70 \mathrm{kOe}$ are obtained for all three samples. Here, we select the data taken at $\mathrm{H}=5 \mathrm{kOe}$ (Fig.1(a),(b),(c)) as representative results. (Detailed measurements of Sample A have been given in Ref. [3]. Additional information about Samples B and C will be presented elsewhere [6].)

The electric field $(E)$ due to the vortex dissipation near the melting transition temperature $\left(T_{M}\right)$ yields the scaling relation $[3,7,8]$ :

$$
E=J\left|1-\left(T / T_{M}\right)\right|^{\nu(2+z-d)} \tilde{E}_{ \pm}(\tilde{J}),
$$

where $\nu$ and $z$ are the static and dynamic exponents, respectively, $\mathrm{d}$ is the dimensionality, $\tilde{E}_{ \pm}(\tilde{J})$ are the universal functions for $T>T_{M}\left(\tilde{E}_{+}\right)$and $T<T_{M}\left(\tilde{E}_{-}\right)$, and $\tilde{J} \equiv(J / T) \mid 1-$ $\left.\left(T / T_{M}\right)\right|^{\nu(1-d)}$. Defining $\tilde{E}=(E / J)\left|1-\left(T / T_{M}\right)\right|^{\nu(d-2-z)}$, we can "collapse" all $E$ vs $J$ isotherms into two universal curves $\tilde{E}_{ \pm}$with proper values of $T_{M}(H), \nu$ and $z$ (See the insets in Fig.1). For both Samples B and C and for all fields, the critical scaling analysis gives $\nu=0.65 \pm 0.05$ and $z=3.0 \pm 0.2$, consistent with those obtained from the $d c$ and $a c$ transport data of Sample A $[3,4]$. Our present results provide evidence of the universal critical scaling behavior in samples with different densities of defects, lending another strong support for a second-order phase transition.

The $T_{M}(H)$ values obtained with the above scaling analysis are plotted in Fig.2. The melting lines for Samples A, B, and C follow the same relation $H_{M}(T)=H_{M}(0) \mid 1-$ $\left.\left(T / T_{c 0}\right)\right|^{2 \nu_{0}}$ with a single zero-field critical exponent $\nu_{0}=0.63 \pm 0.02$. The only difference in the melting lines is the decreasing $T_{c 0}$ values (see Table 1 ). Since $T_{c 0} \sim v_{F} / \xi_{s}$, where $v_{F}$ is the Fermi velocity, and since $v_{F} \propto l$, where $l$ is the mean-free-path, it is clear that the decreasing $l$ with the increasing disorder results in a smaller $T_{c 0}$ value.

Experimentally, the mean-free-path $l$ in a vortex system can be estimated as follows [3]. At constant $T$ and $H$, a characteristic current density $J_{l}(T, H)$ can be defined by the relation $\xi_{v}\left(T, H, J_{l}\right)=l$, where $\xi_{v}$ is the vortex correlation length [3]. Since $J_{l}\left(T_{M}(H)\right)=$ $\frac{k_{B} T_{M}(H)}{l^{2} \Phi_{0}}[3]$ and $J_{l}(T, H)$ can be measured by identifying the current density below which the $\tilde{E}$ vs $\tilde{J}$ scaling relation in Eq. (1) breaks down, we can obtain the vortex meanfree-path $l(H)$. While the $l(H)$ values thus obtained for Sample A are comparable to the average twin boundary separation [3], those for Samples B and C decrease with increasing disorder. The comparison of the $l$ values for Samples A, B and C at $H=5.0 k O e$ is shown in Table 1. 

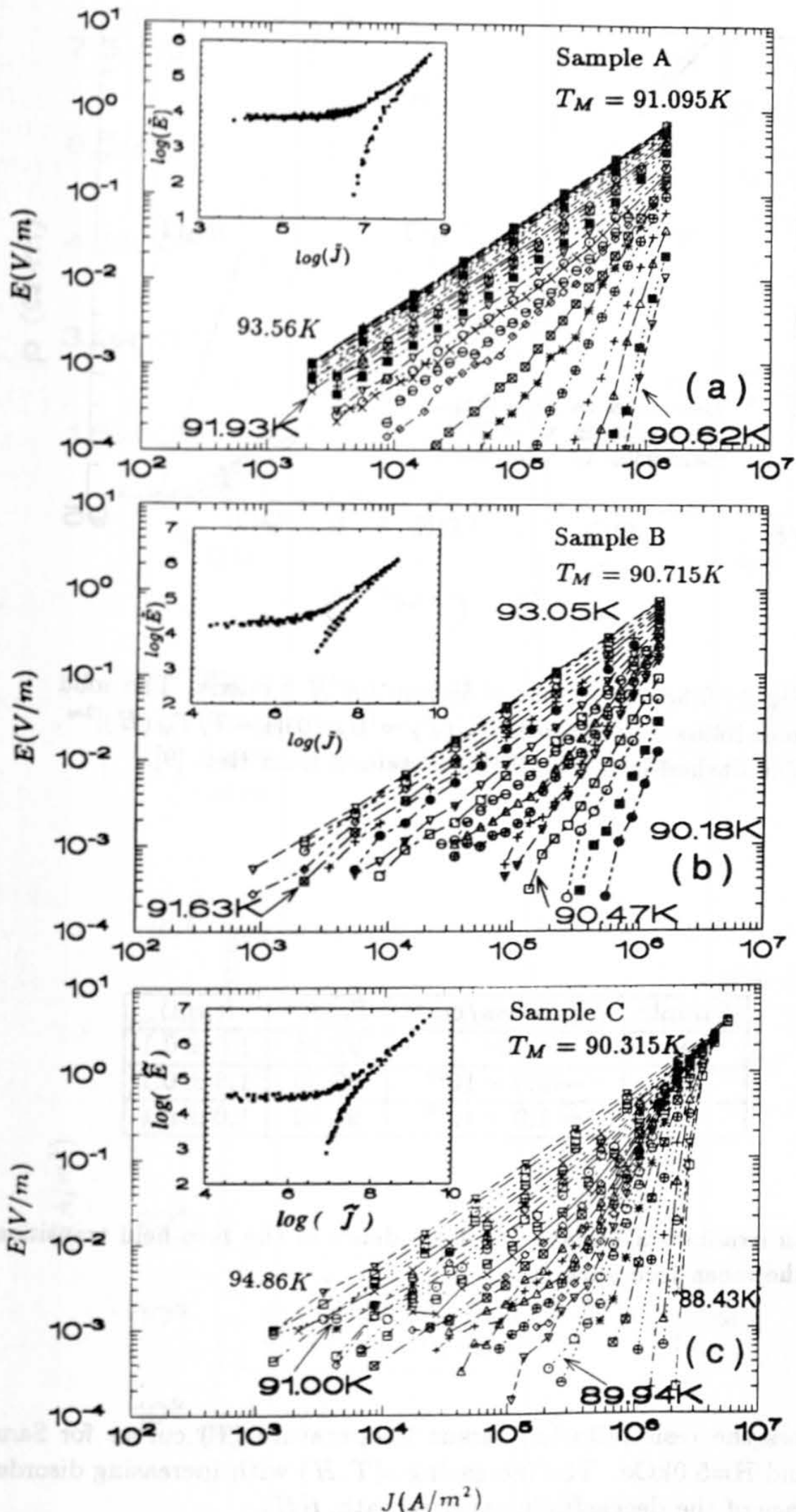

Fig.1. The electric field $(E)$ vs the current density $(J)$ isotherms for $H \| c$-axis and $H=5.0 \mathrm{kOe}$ of the samples (a) without irradiation, (b) irradiated with a fluence of $\sim 5.0 \times 10^{15}$ protons $/ \mathrm{cm}^{2}$, and (c) irradiated with a fluence of $\sim 1.0 \times 10^{16} \mathrm{protons} / \mathrm{cm}^{2}$. The insets are the universal functions $\tilde{E}$ vs $\tilde{J}$ obtained from "collapsing" the isotherms with $J>J_{l}(T, H)$ at the temperatures within the range indicated by the arrows. 


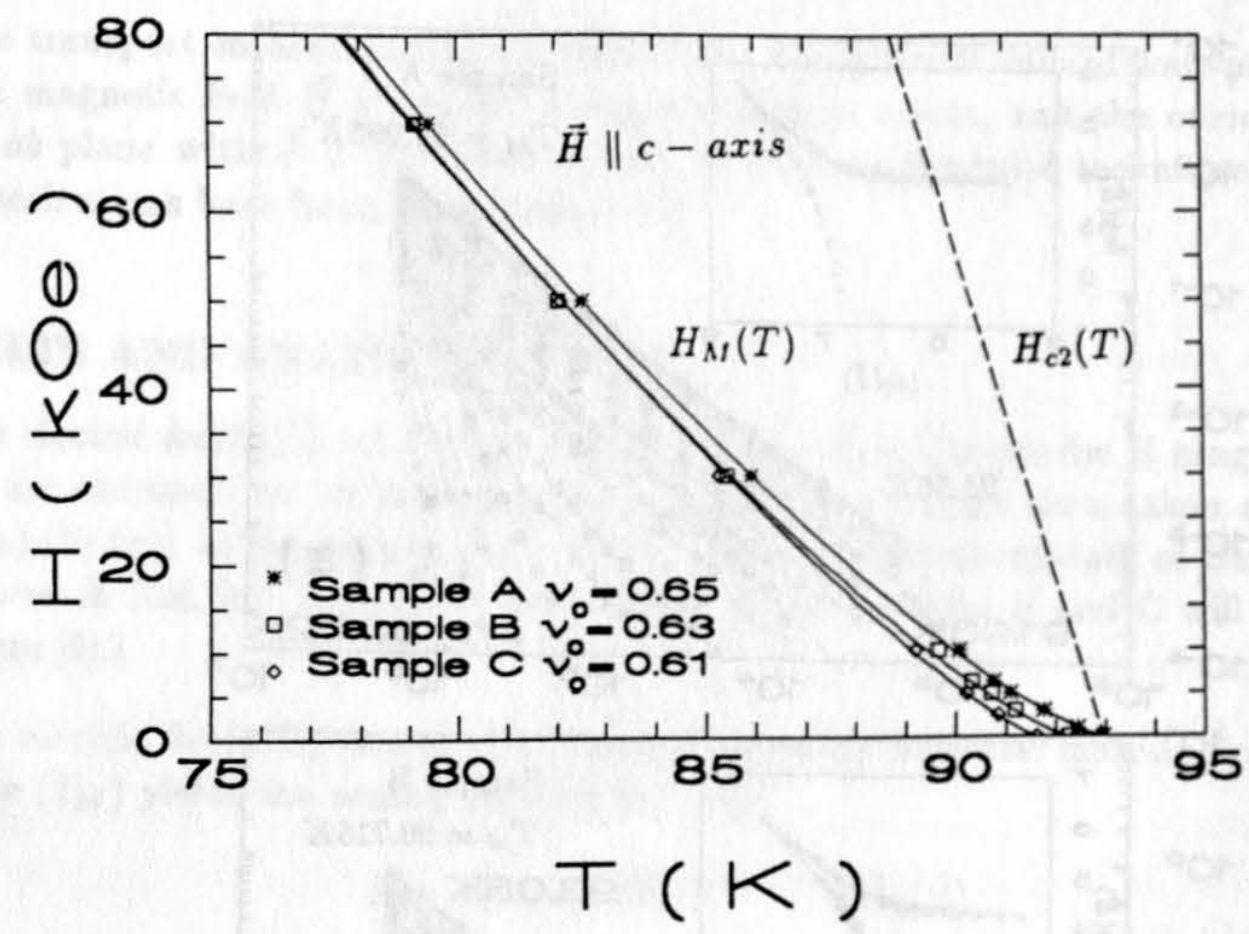

Fig.2. The vortex phase digram for $\vec{H} \| c$-axis. The solid lines follow the relation $H_{M}(T)=H_{M}(0)\left|1-T / T_{M}(H)\right|^{2 \nu}$. The dashed line $\left(H_{c 2}(T)\right)$ is obtained from Ref. [9].

\begin{tabular}{|c|c|c|c|}
\hline Sample & $f$ (protons $\left./ \mathrm{cm}^{2}\right)$ & $T_{\mathrm{c} 0}(K)$ & $l(\mu \mathrm{m})$ \\
\hline A & 0 & 92.95 & $1.6 \pm 0.1$ \\
\hline B & $\sim 5.0 \times 10^{15}$ & 92.25 & $1.3 \pm 0.1$ \\
\hline C & $\sim 1.0 \times 10^{16}$ & 91.62 & $1.0 \pm 0.1$ \\
\hline
\end{tabular}

Table 1. Proton irradiation fluence $(f)$ dependence of the zero-field transition temperature $\left(T_{c 0}\right)$ and the mean-free-path $(l)$.

Figure 3 shows the resistivity $(\rho)$ versus temperature $(T)$ curves for Samples A, B and $\mathrm{C}$ at $\mathrm{H}=0$ and $\mathrm{H}=5.0 \mathrm{kOe}$. The increasing $\rho(T, H)$ with increasing disorder provides additional evidence of the deceasing mean-free-path, $l(H)$.

Finally, we also find evidence of enhanced vortex pinning after irradiations. As shown in Fig.4, the critical current density $J_{c}$ near $T_{c 0}$ (defined with the $2 \mu \mathrm{V} / \mathrm{cm}$ criterion) increases with the increasing proton fluence, consistent with previous reports $[1,2]$ based on magnetization measurements. 


\section{CONCLUSION}

In summary, we have shown evidence of a second-order vortex-solid melting transition in moderately irradiated $\mathrm{Y}-\mathrm{Ba}-\mathrm{Cu}-\mathrm{O}$ single crystals. In addition, we provide quantitative analysis of the decreasing mean-free-path as the result of the increasing defects in the irradiated samples.

\section{ACKNOWLEDGMENT}

We thank Nils Asplund for his technical assistance. This work is jointly supported by ONR Grant \#N00014-91-1556, NASA/OAET, JPL Directory Fund, IBM-Caltech Cooperative Research Fund, and NSF Grant DMR90-11230.

\section{REFERENCES}

[1] L. Civale, A. D. Marwick, M. W. McElfresh, A. P. Malozemoff, and F. Holtzberg. Phys. Rev. Lett. 65, 1164 (1990).

[2] R. B. van Dover, E. M. Gyorgy, L. F. Schneemeyer, A. E. White, S. Glarum, R. J. Felder, and J. V. Waszczak. Mat. Res. Soc. Symp. Proc., 169, 911 (1989).

[3] N.-C. Yeh, W. Jiang, D. S. Reed, U. Kriplani, and F. Holtzberg. Submitted to Phys. Rev. Lett., (April, 1992); Mat. Res. Soc. Symp. Proc. (1992).

[4] D. S. Reed, N.-C. Yeh, W. Jiang, U. Kriplani, and F. Holtzberg. Submitted to Phys. Rev. Lett., (April, 1992); Mat. Res. Soc. Symp. Proc. (1992).

[5] N.-C. Yeh, D. S. Reed, W. Jiang, U. Kriplani, F. Holtzberg, A. Gupta, B.D. Hunt, R.P. Vasquez, M.C. Foote, and L. Bajuk. Phys. Rev. B45, 5654 (1992).

[6] W. Jiang, N.-C. Yeh, D. S. Reed, U. Kriplani, F. Holtzberg, and T. A. Tombrello. in preparation.

[7] N.-C. Yeh, W. Jiang, D. S. Reed, U. Kriplani, F. Holtzberg, and A. Kussumal. Phys. Rev. B45, 5710 (1992).

[8] D. S. Fisher, M. P. A. Fisher, and D. Huse. Phys. Rev. B43, 130 (1991).

[9] U. Welp, W. K. Kwok, G. W. Crabtree, K. G. Vandervoort, and J. Z. Liu. Phys. Rev. Lett. 62, 1908 (1989). 